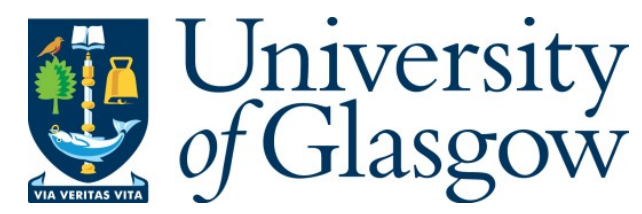

Bernassau, A.L., Al-Rawhani, M ., B eel ey, J ., and Cumming,

D.R.S. (2013) Integrated ultrasonic particle positioning and low excitation light fluorescence imaging. A pplied Physics L etters, 103 (24). p. 244103. ISSN 0003-6951

Copyright $\odot 2013$ A merican Institute of Physics

A copy can be downloaded for personal non-commercial research or study, without prior permission or charge

Content must not be changed in any way or reproduced in any format or medium without the formal permission of the copyright holder(s)

When referring to this work, full bibliographic details must be given

http://eprints.gla.ac.uk/89500/

Deposited on: 08 A pril 2014

Enlighten - Research publications by members of the University of Glasgow http://eprints.gla.ac.uk 


\title{
Integrated Ultrasonic Particle Positioning and Low Power Excitation Light
}

\section{Fluorescence Imaging}

\author{
A.L. Bernassau ${ }^{\mathrm{a}}$, M. Al-Rawhani ${ }^{\mathrm{a}}$, J. Beeley and D.R.S. Cumming \\ School of Engineering, University of Glasgow, Glasgow, G12 8LT, UK
}

A compact hybrid system has been developed to position and detect fluorescent microparticles. It combines a Single Photon Avalanche Diode (SPAD) imager with an acoustic manipulator. The detector comprises a SPAD array, light-emitting diode, lenses and optical filters. The acoustic device is formed of multiple transducers surrounding an octagonal cavity. By stimulating pairs of transducers simultaneously, an acoustic landscape is created causing fluorescent micro-particles to agglomerate into lines. The fluorescent pattern is excited by a low power light-emitting diode and detected by the SPAD imager. Our technique combines particle manipulation and visualization in a compact, low power, portable setup.

The ability to control biological cells and/or micro-particles into specific patterns while simultaneously monitoring and extracting analytical indicators about the state and the physiology of the patterned cells is critical for a wide range of biological studies such as tissue engineering and cell culturing ${ }^{1,2}$. It has been shown that cells can be positioned onto the active area of ion-sensitive field-effect-transistor (ISFETs) integrated dielectrophoretic electrodes $^{3,4}$ for $\mathrm{pH}$ environment monitoring. Quantitative immuno-fluorescent imaging is an alternative approach, as it provides an intracellular indicator of the metabolism and physiology of the cells in response to specific substances ${ }^{5}$. This approach requires a fluorescence microscope incorporating high power excitation source such as a mercury or xenon lamp ${ }^{6}$. An important part of such a microscope is the charge-coupled device (CCD)

\footnotetext{
${ }^{\mathrm{a}}$ Co-first authors
} 
imager, often cooled, that is used to capture the fluorescence images of the patterned particles. To obtain clear images with this system, it is commonly achieved by increasing the intensity of excitation light or the concentration of the fluorophores. A significant drawback of this approach is that it increases the generation of reactive oxygen species such as singlet oxygen that in turn will damage the cell (phototoxicity) and increase the rate of photo bleaching of the fluorophore itself $^{6}$. Furthermore, the physical bulk of commercial fluorescence microscopes and substantial power required for excitation make this approach unsuitable for applications requiring compactness and/or low excitation power.

A significant improvement can be achieved using a Single Photon Avalanche Diodes (SPAD) array, an ultra-sensitive imager capable of detecting emitted fluorescence light at photon counting level, and thus permitting the use of low power excitation sources. In addition, SPADs, unlike CCD based imagers, do not require cooling, and are fabricated in Complementary Metal Oxide Semiconductor (CMOS) technology ${ }^{7,8}$, allowing miniaturization and integration.

In this letter, we demonstrate a compact, low power-excitation, fluorescence imager used in conjunction with an acoustic pattering device. The proposed integration enables pattering and positioning of micro-scale fluorescent particles while at the same time optically recording the patterns formed by the particles using low excitation source. The acoustic positioning element is based on acoustic standing waves and the fluorescence imager based on a $32 \times 32$ SPAD array of detectors.

The block diagram of the complete system is shown in Fig. 1. The upper part of the setup is the acoustic device which employs multiple transducers surrounding an octagonal cavity and mounted by a 3-D micro-positioner; the lower part hosts the detector which consists of the SPAD imager, a fluorescence interference cube, an $\left(18^{\circ}\right)$ viewing angle light-emitting diode (LED) (Optek Technology, USA, OVM4BTX) as an excitation source, 10x 
(Olympus, Japan) infinity corrected objective lens and a convex lens. The fluorescence interference cube incorporates an excitation band pass filter (Semrock, USA; 430-490 nm), a beam splitter (Semrock, USA; $506 \mathrm{~nm}$ ) and a fluorescence band pass filter (Semrock, USA; $513-555 \mathrm{~nm})$.

Overall, the system operates as follows (Fig. 1): When fluorescent particles are placed inside the cavity of the acoustic device, a pair of opposing transducers are stimulated to create a standing wave causing particles to agglomerate at the nodes and form a pattern. In order to detect this pattern optically, a narrow light beam generated by the LED is passed through the excitation filter and reflected by the beam splitter to the objective lens, which focuses the LED's beam onto the fluorescence particles. Fluorescent light emitted by the particles at the surface of the acoustic device is then collected by the objective lens. This light is then passed through the fluorescence filter to a convex lens which in turn focuses the image onto the active area of the SPAD imager.

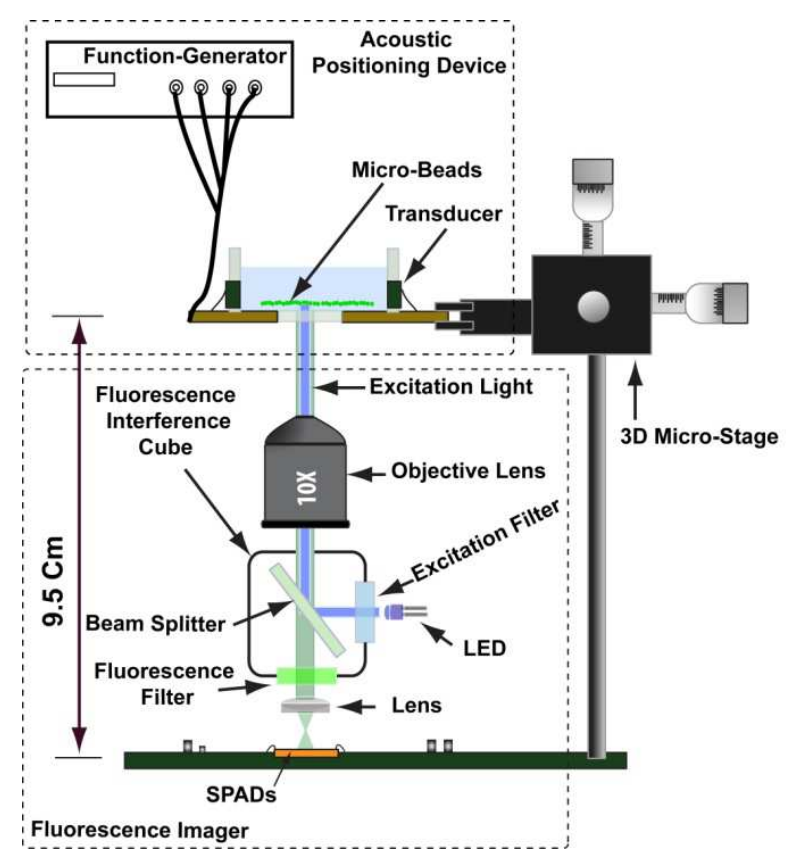

FIG. 1. (Color online) Schematic of the integrated ultrasonic particle positioning and the low excitation light fluorescence imaging. The fluorescent particles are patterned by stimulating the transducers of the acoustic device. The formed patterns are directly monitored and recorded by the fluorescence imaging system. 
The acoustic device (Fig. 2) is formed using eight piezoelectric transducers shaped into an octagonal cavity. Eight $5 \mathrm{~mm} \times 5 \mathrm{~mm}$ plates of (NCE51 Noliac ceramic) lead zirconate titanate (PZT) had an alumina loaded epoxy acoustic matching layer applied. The thickness of the matching layer was optimised using a one-dimensional transmission line model to minimise the reflection of the incident waves ${ }^{9}$. The matching layers reduce resonances that would result in unwanted standing waves with nodal positions that were fixed by the geometry thus inhibiting translation of the field by phase variation. Matched plates without a backing layer were sufficient for trapping and manipulating the micro particles ${ }^{10}$. The matched plates were bonded to a flexible kapton ribbon that was then folded into an octagon. Synchronized transducer drive was achieved using two linked arbitrary waveform generators providing four output channels each (TGA12104, Thurlby Thandar Instruments, UK) allowing independent control of the amplitude, phase and frequency of each channel, controlled by a general purpose interface bus (GPIB) interface using software custom written in Labview. The signals from the waveform generators were amplified and matched by high speed buffers (AD811 Analog Devices USA; BUF634T, Texas Instruments, USA). Patterning of micro-particles was demonstrated both experimentally and by using a computational model to predict the pressure fields. The wave field generated by one transducer, $g(r)$, was modeled as the sum of several cylindrical point sources, $f(r)$ using Huygen's principle:

$$
\begin{aligned}
& f(r)=A e^{\frac{-\alpha r}{\lambda}} \cos (\omega t-k r+\Phi) \\
& g(r)=\sum_{i=1}^{n} f\left(r_{i}\right)
\end{aligned}
$$

where $A$ is the amplitude, $\alpha$ is the damping factor, $\lambda$ is the wavelength, and $\Phi$ is the initial phase. The device boundaries were assumed to be perfectly absorbing. 


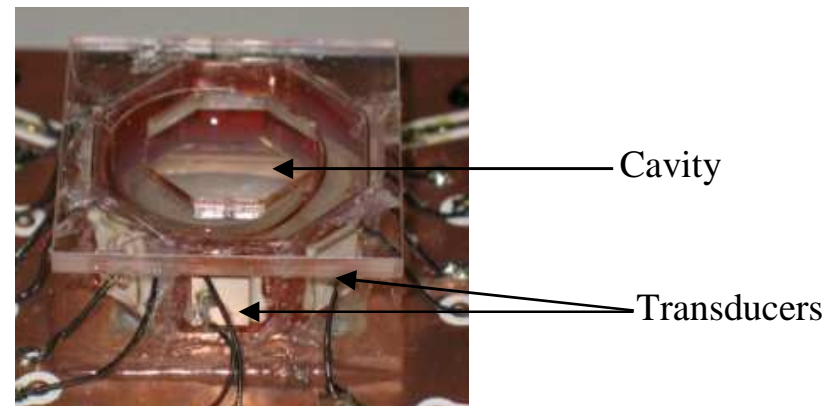

FIG. 2. (Color online) Photograph of the octagonal acoustic manipulator.

The key component in the fluorescence detector part of the system is an applicationspecific integrated circuit (ASIC) imager based on a 32x32 SPAD array (Fig. 3a). The ASIC was fabricated according to our own design using a high voltage $0.35 \mu$-CMOS process from Austriamicrosystems (Germany). The SPAD is an avalanche photodiode reverse-biased above its breakdown voltage to operate in Geiger Mode and thus generates pulses proportional to the number of photons incident upon the active area of each $\operatorname{SPAD}^{7}$.

The physical structure of the SPAD fabricated in this chip is based on diffused guard ring junction as illustrated in Fig. 3b. Since the CMOS process is a planar fabrication process, the p-n junction has small curvature around its edges which results in higher electric field in the edges of the p-n junction than the main active area. As a result, in high fields near to avalanche breakdown, the edges will breakdown at lower voltages than the main area. To avoid this premature breakdown, a guard ring of a lower doped material, the same as the anode, is diffused around the edges of the active area. The lower doped material reduces the electric field at the edges hence preventing premature breakdown. Similarly, as the typical layout of a p-n junction is rectangular, higher fields occur in the corners. In order to avoid these high electrical fields in the corners, the SPAD has a circular layout creating a smooth edge hence generating a uniform electric field across the active area. 


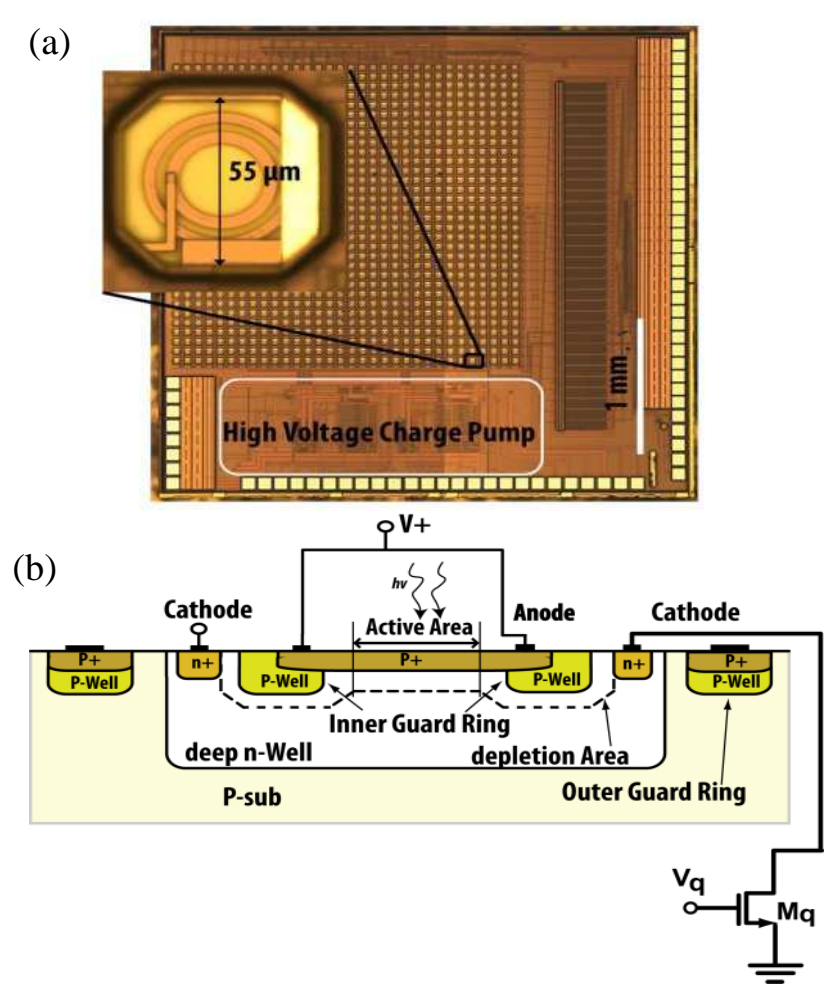

FIG. 3. (a) Photograph of the $3.7 \mathrm{~mm} \times 3.7 \mathrm{~mm}$ ASIC chip. The imager active area is $2.4 \mathrm{~mm} \times 2.4 \mathrm{~mm}$. The ASIC incorporates: 32x32 SPAD pixels, controllable charge pump (3-37.9 V), column multiplexer, 32x (16-bit) digital pulse counters and readout multiplexer. (b) Cross section through the center of a circular SPAD showing the guard ring of low doped material used to reduce the electric field at the edges and corners. For each SPAD the active area where the measurement take place is $24 \mu \mathrm{m}$ diameter, and the overall area including the guard ring is $55 \mu \mathrm{m}$ in diameter.

The ASIC uses a "rolling shutter" readout arrangement, in which one 32-pixel column at a time is powered via a column multiplexer, and the pulse output from the column's SPADs during a defined counter gate period is counted by an array of 32 16-bit counters. A row multiplexer then permits serial readout of pulse counts from the counters. The ASIC operation has been evaluated experimentally. The SPAD's Photon Detection Efficiency (PDE) spans between blue-green region and has a peak of $42.4 \%$ at $475 \mathrm{~nm}$ when biased at 3.1 $\mathrm{V}$ above its $18.9 \mathrm{~V}$ breakdown voltage. Under dark conditions the average dark count of the SPAD is c. $7 \mathrm{kcps}$. Fig. 4a shows a grey scale representation of the dark count rates of the 
SPAD array. At high illumination a single SPAD of the 1024 SPADs in the array can generate up to $4000 \mathrm{kcps}$ before it reaches saturation. The ASIC is powered by a $3 \mathrm{~V}$ power supply and draws a maximum current of $1.74 \mathrm{~mA}$.

Prior to using the imaging system to detect patterned fluorescent particles, we determined the minimum fluorescent particle concentration that can be detected by the SPAD imager. This operating parameter was set by choosing a bias point for the LED that gave an average count rate twice the dark count. In practice, this was achieved by trying different concentrations of $10 \mu \mathrm{m}$ diameter fluorescent polystyrene particles (Polysciences Europe, Germany). At LED bias point $2.6 \mathrm{~V}(3.6 \mathrm{~mA})$, measurements in Fig. $4 \mathrm{~b}$ shows that the imager can efficiently detect a minimum of $1.3 \times 10^{6} \pm 10 \%$ particles $/ \mathrm{mL}$ and produces more than $21.5 \mathrm{kcps}$ which is more than twice the average dark count. 
(a)
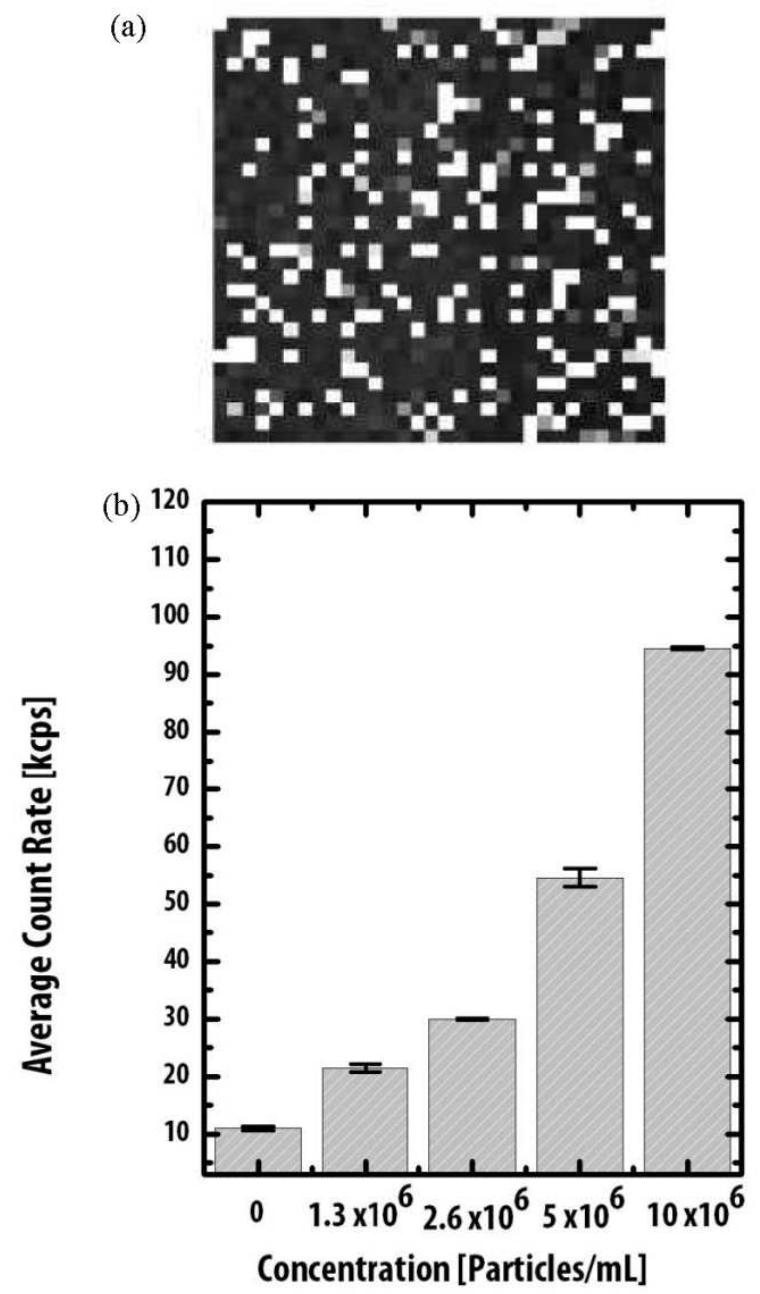

FIG. 4. (a) Grey scale image from the SPAD array in the dark showing the fixed pattern noise (b) a graph of the average count rate from the SPADs in the array as a function of particle concentration in the suspension place into the acoustic system with no stimulation. The inner graph shows the time response of a single SPAD to fluorescence emission.

Patterning experiments were performed by stimulating the acoustic transducers with a 4.00 MHz sine-wave and a $8 \mathrm{~V}_{\mathrm{pp}}$ amplitude. At this frequency, the wavelength of the sound waves in water was $\lambda=375 \mu \mathrm{m}$. By using a relatively low fluorescent concentration of $2.6 \times 10^{6} \pm 10 \%$ particles $/ \mathrm{mL}$ and biasing the LED excitation source at $2.6 \mathrm{~V}(3.6 \mathrm{~mA})$, we were able to detect the formed patterns on the cavity of the acoustic device reliably. Fig. 5 (left hand side) shows the computer simulation results of the acoustic landscape obtained with two transducers at different orientation and the corresponding experimental results (right hand side). For two active transducers, a linear pattern of nodes and antinodes is formed. The 
distance $d$ between the nodes is $d=188 \mu \mathrm{m} \pm 10 \%$, as expected. It can be seen from the computational results in Fig. 5 that regular standing wave patterns are achieved in the middle of the octagonal cavity at the intersection of the travelling waves. The micro-particles are trapped at the minima of the potential acoustic energy density ${ }^{11}$. It can be seen that the patterning of the trapped particles experimentally follows this behavior.

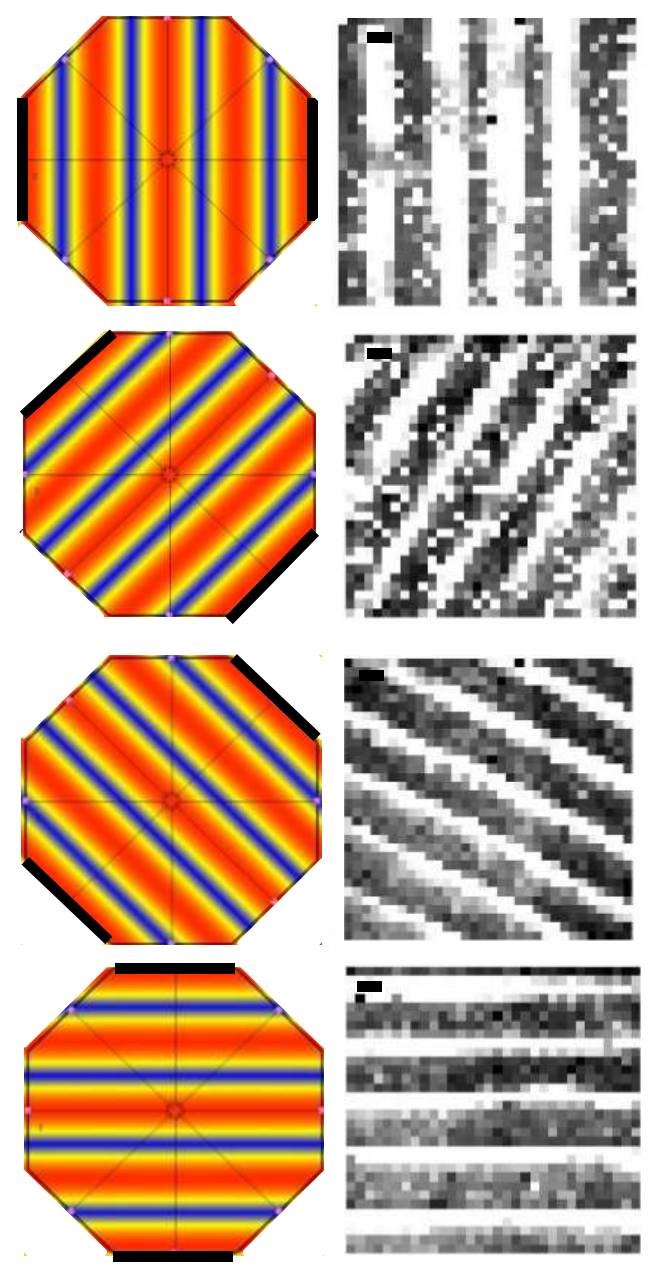

FIG. 5. (Color online) Computer simulations and experimental observations of the acoustic landscape obtained with two transducers when they are excited in phase. For each figure the simulation is on the left and the experimental results using $10 \mu \mathrm{m}$ fluorescent polystyrene particles is on the right. The active transducers are shown in black bold line on the simulation. In the simulation the acoustic potential energy maxima are in red, and the acoustic energy minima are in blue. (Scale bar = $100 \mu \mathrm{m}$ for the right-hand side images). 
In conclusion, we have presented a compact, low power-excitation, fluorescence imager used in conjunction with acoustic pattering. The integrated system has been shown to pattern micro-fluorescence particles and simultaneously monitor and record the formed pattern using SPAD array imager. The high sensitivity of the SPAD array allows fluorescence imaging to be performed using a low power LED excitation source. The acoustic standing wave concentrates the particles in patterns that can be more easily be detected. Single cell imaging can be achieved by changing the objective lens to a higher magnification. This proof of concept system opens the door for applications that require fluorescence imaging of pattering processes of living biological cells such as cell engineering and culture.

1 A. Bernassau, F. Gesellchen, P. MacPherson, M. Riehle, and D. Cumming, Biomedical Microdevices 14, 559-564 (2012).

2 B. Vanherberghen, O. Manneberg, A. Christakou, T. Frisk, M. Ohlin, H. M. Hertz, B. Onfelt, and M. Wiklund, Lab on a Chip 10, 2727-2732 (2010).

3 M. Castellarnau, N. Zine, J. Bausells, C. Madrid, A. Juarez, J. Samitier, and A. Errachid, Sensors and Actuators B: Chemical 120, 615-620 (2007).

4 M. Castellarnau, N. Zine, J. Bausells, C. Madrid, A. Jua;rez, J. Samitier, and A. Errachid, Materials Science and Engineering: C 26, 405-410 (2006).

5 M. Nakao, S. Inoue, R. Oishi, T. Yoshinobu, and H. Iwasaki, Journal of Fermentation and Bioengineering 79, 163-166 (1995).

6 T. Nishigaki, C. D. Wood, K. Shiba, S. A. Baba, and A. Darszon, Biotechniques 41, 191-197 (2006).

G. F. D. Betta, L. Pancheri, and D. Stoppa, IEEE Lasers and Electro-Optics Society, 354-355 (2008).

M. A. Al-Rawhani, D. Chitnis, J. Beeley, S. Collins, and D. R. Cumming, IEEE Trans Biomed Eng. 60, 55-62 (2013).

C. R. P. Courtney, C. K. Ong, B. W. Drinkwater, A. L. Bernassau, P. D. Wilcox, and D. R. S. Cumming, Proceedings of the Royal Society A: Mathematical, Physical and Engineering Science 468, 337-360 (2012).

10 A. L. Bernassau, C. R. P. Courtney, J. Beeley, B. W. Drinkwater, and D. R. S. Cumming, Applied Physics Letters 102, 164101 (2013).

11 A. Bernassau, P. MacPherson, J. Beeley, B. W. Drinkwater, and D. Cumming, Biomedical Microdevices 15, $289-297$ (2013). 